\title{
Cancer Transcriptome Dataset Analysis: Comparing Methods of Pathway and Gene Regulatory Network-Based Cluster Identification
}

\author{
Seungyoon Nam ${ }^{1-3}$
}

\begin{abstract}
Cancer transcriptome analysis is one of the leading areas of Big Data science, biomarker, and pharmaceutical discovery, not to forget personalized medicine. Yet, cancer transcriptomics and postgenomic medicine require innovation in bioinformatics as well as comparison of the performance of available algorithms. In this data analytics context, the value of network generation and algorithms has been widely underscored for addressing the salient questions in cancer pathogenesis. Analysis of cancer trancriptome often results in complicated networks where identification of network modularity remains critical, for example, in delineating the "druggable" molecular targets. Network clustering is useful, but depends on the network topology in and of itself. Notably, the performance of different network-generating tools for network cluster (NC) identification has been little investigated to date. Hence, using gastric cancer (GC) transcriptomic datasets, we compared two algorithms for generating pathway versus gene regulatory network-based NCs, showing that the pathway-based approach better agrees with a reference set of cancer-functional contexts. Finally, by applying pathway-based NC identification to GC transcriptome datasets, we describe cancer NCs that associate with candidate therapeutic targets and biomarkers in GC. These observations collectively inform future research on cancer transcriptomics, drug discovery, and rational development of new analysis tools for optimal harnessing of omics data.
\end{abstract}

Keywords: cancer transcriptomics, computational biology, gastric cancer, genomics, systems biology

\section{Introduction}

$\mathbf{T}$ RANSCRIPTOME ANALYSIS IS BROADLY ADVOCATED for biomarker discovery, personalized medicine, and functional understanding of complex biological systems in health and disease states such as cancer (Karagoz et al., 2015; Mirsafian et al., 2016; Waldron and Riester, 2016). However, such analysis often faces considerable complexity, due to interdependencies between gene entries.

Networks from transcriptome datasets reveal regulatory relationships among biological entities, providing a systems scale understanding of molecular mechanisms (Barabasi and Oltvai, 2004). The importance of network generation has been widely accepted for addressing biological questions, including those in cancer pathogenesis (Aytes et al., 2014), and distinct algorithms have also been introduced to study networks. Broadly, there are two types of algorithms: signaling pathway networks (SPNs) and gene regulatory networks (GRNs). While many comparisons between GRN-generating algorithms have been performed, such comparisons between SPNs and GRNs, in terms of network clusters (NCs), have been little evaluated.

Network clustering provides clues for capturing important regions within complex network topologies, in terms of densely connected regions (i.e., "clusters") (Morris et al., 2011). To obtain such clusters from high-throughput "omics" data, different frameworks can be used. While the SPN framework utilizes both prior signaling knowledge and publically available omics data, GRN uses only omics data, without prior information. Further transformation of networks (from these two frameworks) into clusters has not yet been attempted, in terms

\footnotetext{
${ }^{1}$ Department of Genome Medicine and Science, College of Medicine, Gachon University, Incheon, Korea.

${ }^{2}$ Department of Life Sciences, Gachon University, Seongnam, Korea.

${ }^{3}$ Gachon Institute of Genome Medicine and Science, Gachon University Gil Medical Center, Incheon, Korea.
}

(c) Seungyoon Nam, 2016. Published by Mary Ann Liebert, Inc. This Open Access article is distributed under the terms of the Creative Commons License (http://creativecommons.org/licenses/by/4.0), which permits unrestricted use, distribution, and reproduction in any medium, provided the original work is properly credited. 
of which approach provides more informative NCs having cancer-related functional contexts.

Knowledge of intracellular oncogenic signal transduction reveals potential "druggable" molecular targets (Jia et al., 2009). Consequently, targeted cancer therapy requires a priori understanding of the molecular mechanisms involved in tumor pathogenesis (Barabasi and Oltvai, 2004; Berger and Iyengar, 2009; Jia et al., 2009). Since a network derived from highthroughput ("omics") technologies involves a highly complex set of molecular mechanisms (Barabasi and Oltvai, 2004), it is not computationally feasible to incorporate all the signaling data from a particular network, for determining potential therapeutic strategies. Thus, these data must be "narrowed down" into subsets of molecular mechanisms, represented as NCs. As expected, this filtering process assumes that densely connected regions, or NCs, converge at functional "hubs" that may subsequently align with potential carcinogenic molecular mechanisms (Nam et al., 2012), for the potential discovery of effective "targeted" therapeutics (Barabasi and Oltvai, 2004; Berger and Iyengar, 2009; Goymer, 2008).

However, despite these promising approaches, it has yet to be demonstrated whether SPN or GRN methods yield more reliable NCs (in terms of cancer-functional contexts) (Morris et al., 2011). In this study, we compared our previously developed algorithm, PATHOME ( pathway and transcriptome information) (Nam et al., 2014), as an SPN method, with ARACNE (Algorithm for the Reconstruction of Accurate Cellular Networks) (Margolin et al., 2006), as a GRN method, in terms of agreement between NCs with a reference set (Futreal et al., 2004) of cancer-related functional contexts. The results of this comparison indicated that NCs of PATHOME, compared to those of ARACNE, better aligned with the reference set of cancer-functional contexts. We specifically used gastric cancer (GC), the fourth most worldwide common cancer type (Chang et al., 2016), as an example disease having few effective targeted therapies, due to limited understanding of its underlying biological bases (in terms of delineating network biology and clusters).

In sum, we applied PATHOME, and a network-clustering algorithm (Morris et al., 2011), to derive GC networkderived clusters (and potentially important therapeutic targets), in addition to improved mechanistic understanding of GC etiology. Also, the new observations reported in this study collectively inform future research on cancer transcriptomics, drug discovery, and rational development of new analysis tools for optimal harnessing of omics data.

\section{Materials and Methods}

\section{Transcriptomic datasets}

For comparing NCs, we obtained $3 \mathrm{GC}$ RNA-Seq and microarray transcriptomic datasets, GEO (www.ncbi.nlm.nih.gov/ geo) accessions GSE37023 (Wu et al., 2013), consisting of 112 GC tumors and 39 normal tissues; GSE36968 (Kim et al., 2012), containing $24 \mathrm{GC}$ tumors and 6 noncancerous specimens; and GSE27342 (Cui et al., 2011), comprising 80 GC tumor samples and paired normal tissues (Table 1). These three datasets were used for constructing networks, as described below.

\section{Construction of PATHOME and ARACNE networks}

We first used "PATHOME” (Nam et al., 2014) to generate SPNs (henceforth, "PATHOME networks") from analyzing the three transcriptomic datasets, using the default option $(p<0.05)$. Obtaining genes retained in the PATHOME network, for each dataset, we applied ARACNE (Margolin et al., 2006) to the matrix of expressed genes, to generate GRNs (henceforth, "ARACNE networks") having different connections between the genes. In application of ARACNE, we used its default options.

\section{PATHOME-derived NCs versus ARACNE-derived NCS}

For each dataset, NCs, from PATHOME and ARACNE networks, were identified using the Markov Clustering algorithm (MCL), implemented in clusterMaker (Morris et al., 2011). Using the NCs obtained by the two approaches, we performed two comparisons: first, whether the PATHOME or ARACNE networks contained more NCs; and second, we compared whether NCs derived from PATHOME networks (henceforth, "PATHOME-NCs") or NCs derived from ARACNE networks (henceforth, "ARACNE-NCs") contained more cancer-related functional contexts, represented matches to a set of Gene Ontology (GO) terms.

In the first comparison, since NCs represent functional "hubs" (i.e., convergence points for multiple pathways) (Barabasi and Oltvai, 2004; Berger and Iyengar, 2009; Goymer, 2008), the number of NCs included in any specific network is important. In the first comparison, we compared the number of PATHOME- and ARACNE-NCs (defined above), for all three transcriptome datasets. We further examined the obtained number of NCs, according to the total number of $\mathrm{NC}$ entries, using the two approaches.

For the second comparison, we set up a reference set of cancer-functional contexts associated with specific GO terms. By inputting the gene list (Futreal et al., 2004) of the cancer Gene Census repository (cancer.sanger.ac.uk/cosmic/ census) into DAVID v6.7 (Huang et al., 2007) (under a significance cutoff as default EASE (Huang et al., 2007) score threshold of 0.1 ), we obtained 973 GO terms significantly associated with the gene list. The 973 GO terms (henceforth, reference GOs) were set as the reference set of cancer-related functional contexts, including various cancer "hallmark" (Hanahan and Weinberg, 2011).

Table 1. Three Public Gastric Cancer Datasets in the Study

\begin{tabular}{llll}
\hline GEO accession & & \multicolumn{1}{c}{ Description } & Experiment \\
\hline GSE37023 & Gastric tumors vs. nonneoplastic gastric mucosa & Microarrays & Wu et al. (2013) \\
GSE36968 & Gastric tumor samples vs. noncancerous gastric tissue samples & RNA-SEQ & Kim et al. (2012) \\
GSE27342 & Paired tumor and adjacent nonneoplastic tissues from GC patients & Microarrays & Cui et al. (2011) \\
\hline
\end{tabular}

We used the three GC transcriptome datasets for comparing network cluster identifications and describing GC network clusters. $\mathrm{GC}$, gastric cancer. 
To obtain significant reference GO terms associated with the PATHOME-NCs of a dataset, we only considered NCs having more than four genes. Collecting all the genes from such NCs, we fed the genes into DAVID (under a significance cutoff as default EASE (Huang et al., 2007), score threshold of 0.1), acquiring the number of significant GO terms. We used the default background, provided by DAVID, for the GO assessment.

We then assessed intersections between the significant GO terms of the PATHOME-NCs and the reference GOs, obtaining the number of cancer-functional contexts in PATHOME-NCs. The intersections correspond to the number of detected reference GOs. The same procedure was then repeated for obtaining and examining the number of cancer-functional contexts in ARACNE-NCs. Then, we compared the number of detected reference GOs between ARACNE-NCs and PATHOME-NCs, as reference cancer-related functional contexts.

\section{Results}

Topological difference between PATHOMEand ARACNE-derived networks

A schematic of our approach, using the PATHOME and ARACNE algorithms (Morris et al., 2011), is shown in Figure 1. Briefly, our objective was to compare how the two different network construction methods affected the identification of NCs (Fig. 1), based on each method's topologies. After network construction, we applied a Markov Clustering algorithm (MCL) (Morris et al., 2011) for identifying clusters (Fig. 1). We then inspected which method better reported a reference set of cancer-functional contexts (Fig. 1).
First, we characterized the network topological parameters [clustering coefficient, network centralization, network density, network diameter, network heterogeneity, and network radius; see descriptions in Supplementary Table S1 (Doncheva et al., 2012)] of the two methods, using the three abovementioned datasets (Supplementary Fig. S1 and Supplementary Table S1) and the Cytoscape Network Analyzer plugin (Assenov et al., 2008). In terms of network diameter and heterogeneity, PATHOME (Nam et al., 2014) networks were larger than those of ARACNE (Margolin et al., 2006), for the three datasets (Supplementary Fig. S1 and Supplementary Table S2). With regard to network radii, ARACNE networks were larger than PATHOME networks, in the three datasets (Supplementary Fig. S1), while the clustering coefficients of PATHOME networks and ARACNE networks were nearly identical in two of the datasets [GSE27342 (Cui et al., 2011) and GSE36968 (Kim et al., 2012; Nam et al., 2014)], but differed for GSE37023 (Wu et al., 2013) (Supplementary Fig. S1).

Of additional interest, we observed that the GSE27342 and GSE36968 datasets showed similar patterns of all topological parameters, while GSE37023 (Wu et al., 2013) showed opposite patterns for clustering coefficient and network centralization (Supplementary Fig. S1), implying a different data context for the dataset GSE37023 compared to the other two datasets.

\section{Comparison between PATHOME- and ARACNE-derived NCs}

We next characterized the PATHOME- and ARACNENCs. Since topologically densely connected regions are often regarded biologically critical cascades (Goymer, 2008;

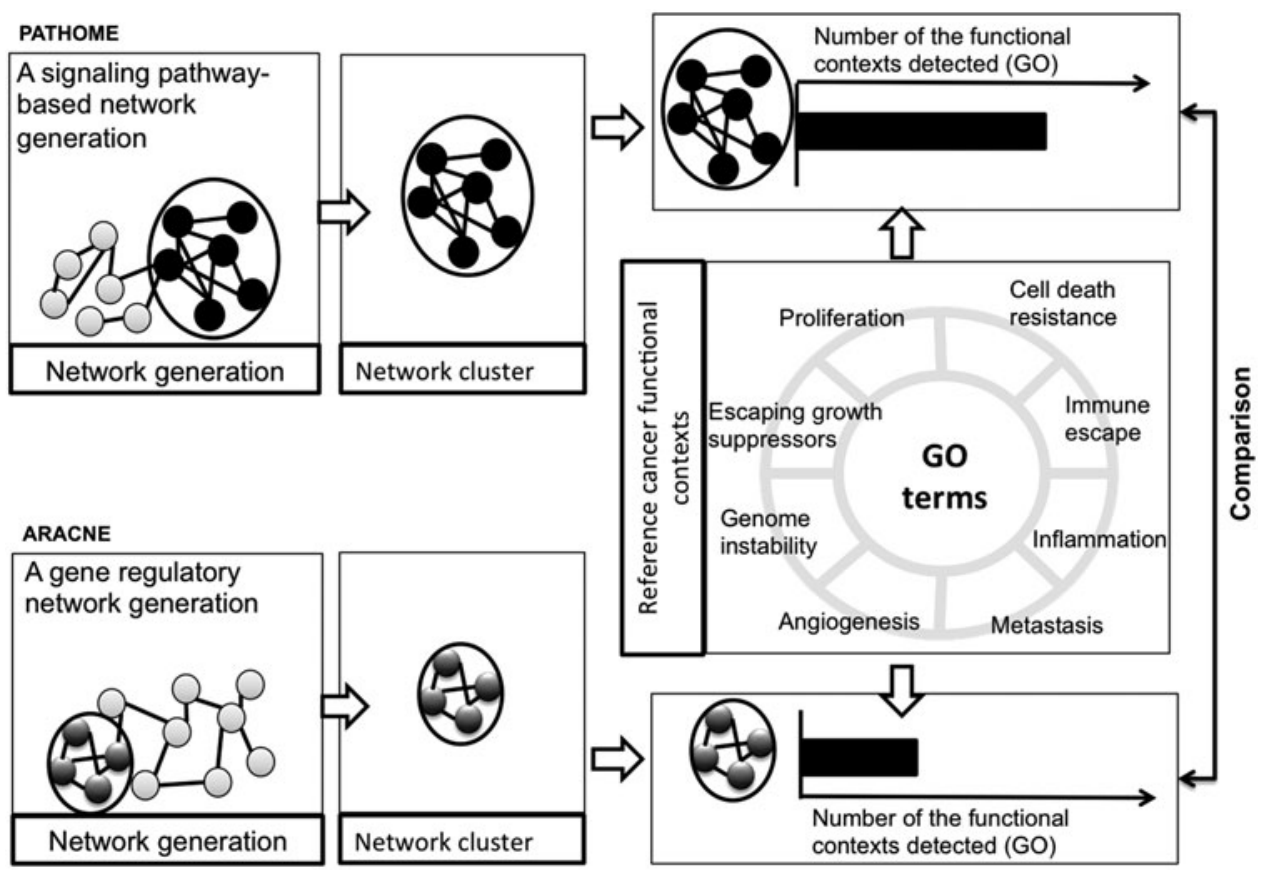

FIG. 1. Overview of the study design and comparisons between PATHOME-NCs and ARACNE-NCs in analysis of three GC transcriptome datasets. Network clustering results depend on network generation methods. In this study, we compared the performance of two differently generated NCs, (PATHOME-NCs and ARACNE-NCs), using the algorithm MCL (Morris et al., 2011). Using GC datasets as examples, we measured whether PATHOME-NCs or ARACNE-NCs detected more cancer-related functional contexts, in terms of cancer-associated GO terms (equivalently, reference GOs). GC, gastric cancer; MCL, Markov Clustering algorithm; GO, Gene Ontology; NC, network cluster. 
Morris et al., 2011), strongly associated with modes of disease mechanisms, acquisition of more NCs from a network is a meaningful comparison for the identification of candidate therapeutic targets (Berger and Iyengar, 2009). In our study, densely connected regions (in networks) refer to NCs identified by the network-clustering algorithm, MCL (Morris et al., 2011). Consequently, using MCL (Morris et al., 2011), we extracted densely connected regions from networks, obtaining more PATHOME-NCs than ARACNE-NCs, from the three real biological datasets: specifically, 81 versus 3, for GSE36968 (Kim et al., 2012; Nam et al., 2014); 81 versus 46, for GSE27342 (Cui et al., 2011); and 88 versus 57, for GSE37023 (Wu et al., 2013) for PATHOME- and ARACNENCs, respectively (Supplementary Table S3).

Therefore, PATHOME-NCs may reveal higher levels of pathway signaling associated with potential therapeutic targeting options, when compared to ARACNE. Also, further dissecting the number of NCs, according to the number of NC entries, PATHOME-NCs reported more NCs with entries (or nodes) greater than or equal to 5, when compared to ARACNE-NCs (Fig. 2A). This indicates that PATHOMENCs report NCs in a collective, but not fragmented, manner.

\section{Comparing PATHOME- and ARACNE-derived cancer-associated NCs}

In addition to the previously described analyses, to identify whether PATHOME- or ARACNE-NCs detected more cancer-related functional contexts, we set reference GO terms significantly associated with the cancer Gene Census (Futreal et al., 2004) as the reference set of cancer-functional contexts (reference GOs; see Materials and Methods section in detail).

In the dataset GSE27342, PATHOME-NCs reported 1098 statistically significant GO terms, with 603 (Fig. 2B) overlapping with cancer reference GOs. In the same dataset, ARACNE-NCs reported five significant GO terms, two (Fig. 2B) of which matched the reference GOs. In dataset GSE37023, by contrast, PATHOME-NCs reported 612 significant GO terms, 404 of which overlapped reference GOs, while ARACNE-NCs reported 451 significant GO terms, with 292 overlapping the reference GOs (Fig. 2B). In dataset GSE36968, ARACNE-NCs, having at least five nodes, were not found, resulting in no GO terms; whereas PATHOMENCs having at least five nodes reported 1063 significant GO
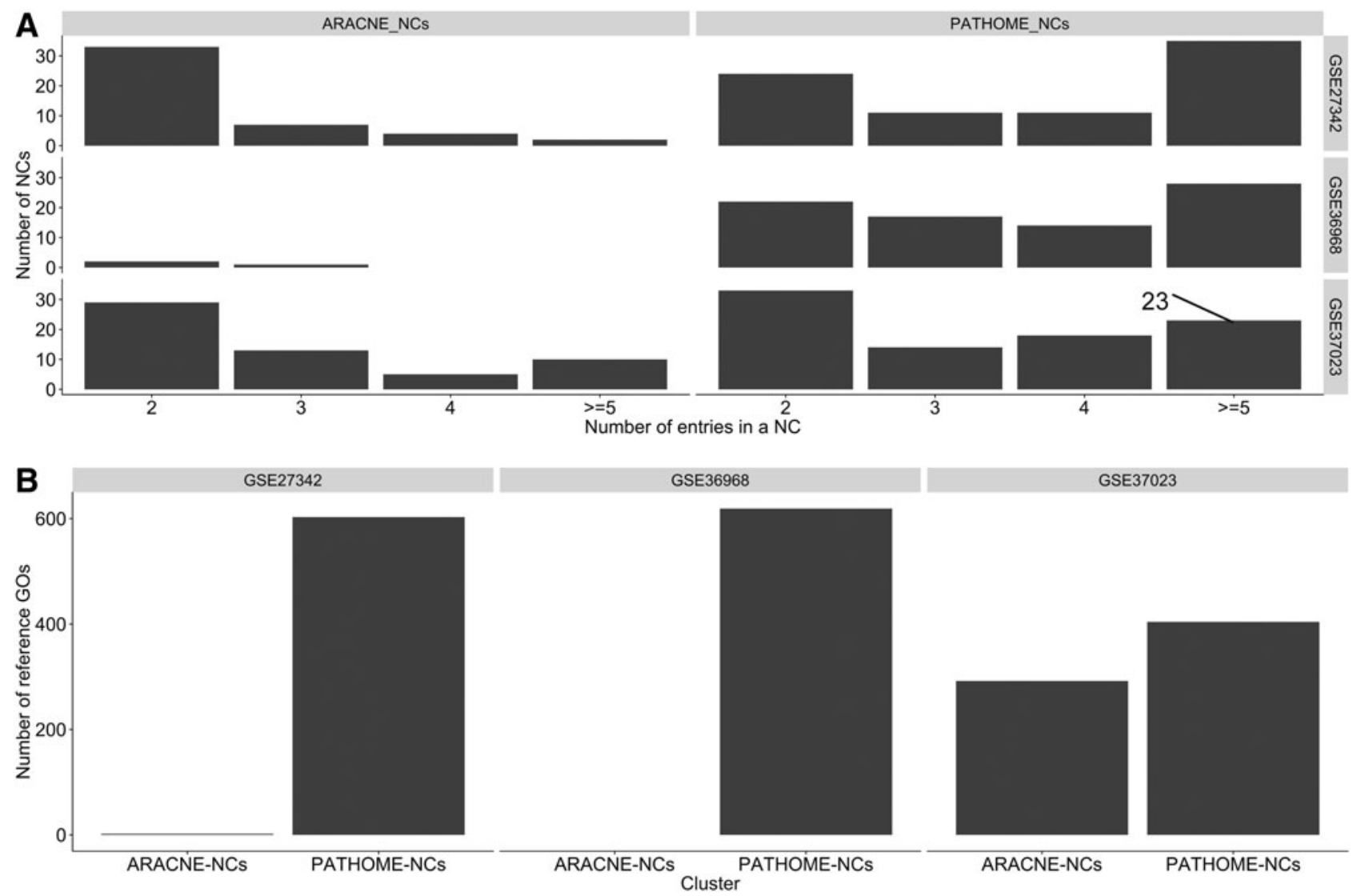

FIG. 2. Comparisons between PATHOME-NCs and ARACNE-NCs in three GC transcriptome datasets. (A) $x$-axis represents the number of entries in specific NCs, while the $y$-axis represents the number of NCs. For example, in the GSE37023 dataset, the number of PATHOME-NCs, having five or more gene entries, was 23 (indicated by the black line). As a whole, PATHOME-NCs had more NCs having cluster entries $\geq 5$, compared to ARACNE-NCs, as derived from the three datasets. These results indicate that PATHOME networks were fragmented into larger gene entry sizes of NCs, compared to ARACNE networks, when the same MCL was applied. (B) In the three GC datasets, the graph represents whether PATHOME-NCs or ARACNE-NCs detected (or contained) more reference cancer-functional GO terms ( $y$-axis). For example, in GSE37023, PATHOME-NCs detected 404 reference cancer-functional GO terms, and ARACNE-NCs detected 292 reference GO terms. 
terms, with 620 of these overlapping the reference GOs (Fig. 2B). Thus, PATHOME-NCs better matched the cancerfunctional contexts, compared to ARACNE-NCs, throughout the comparisons, using the three independent datasets. We confirmed that PATHOME network clustering showed better cancer network identification performance compared to ARACNE. We describe several PATHOME-NCs from the three datasets in the Discussion section.

\section{Discussion}

There is a growing need for innovation in data analysis algorithms and bioinformatics in the context of cancer transcriptomics. As shown in the Supplementary Figure S1, topology parameter differences between PATHOME and ARACNE networks indicate different connectivity patterns. In particular, network diameters (the longest distance between any two nodes) of PATHOME network were consistently longer than those of ARACNE network. This may partly be due to PATHOME's consideration of paths consisting of consecutive edges of more than two nodes (Nam et al., 2014), while ARACNE only considers single edges (without regard to consecutive single edges).

GC, with a worldwide incidence of 952,000 cases and 723,000 deaths (Torre et al., 2015), currently has few nonsurgical therapeutic options (Bang et al., 2010). Toward improving these dire statistics, acquisition of GC-related NCs (as we performed in this study) could facilitate the identification of druggable signaling cascades/networks (Fig. 3).

The NC A was the largest NC ascertained from GSE36968 (transcriptome dataset of Asian GC tumors vs. noncancerous tissues), implicating multiple STAT proteins and JAK family kinases related to immune response and hematopoiesis (Ubel et al., 2013). In this specific cluster, the JAK family kinase genes $T Y K 2$ and JAK2 (among others) were upregulated in GC, compared to normal, tissues. Upregulation of JAK members has been well reported in breast, prostate, and cervical cancers, playing diverse roles in differentiation and cancer cell proliferation and survival (Rane and Reddy, 2000). Moreover, it was recently reported that JAK/STAT pathways upregulate programmed death receptor ligand 1 (PD-L1) in cancer cells, an event that leads to evasion of T cell-mediated antitumor immune responses (Ohaegbulam et al., 2015; Ritprajak and Azuma, 2015). Thus, this cluster suggests the feasibility of immunotherapy in GC as a new therapeutic option, considering that GC has very limited effective targeted therapies (Bang et al., 2010).

In addition to NC A, NC B (as derived from GSE36968; Fig. 3) showed upregulation of the proto-oncogenes $K D R$, FLT1, EGFR, and MET in GC tumors, compared to normal gastric tissues. Of these, KDR and FLT1 are both receptors for VEGFA, the predominant mediator of angiogenesis in tumor progression (Ferrara and Adamis, 2016; Slattery et al., 2014). Thus, this finding links GC to VEGF signaling, a common therapeutic target, in numerous other advanced cancer types, over the last decade (Ferrara and Adamis, 2016; Goel and Mercurio, 2013).

Moreover, tumor-synthesized VEGF is an important immunosuppressive cytokine for eluding immunosurveillant cells (e.g., NK cells, T cells, and so on) (Dunn et al., 2006; Matsueda and Graham, 2014; Nam and Park, 2012), and MET and EGFR are also strong therapeutic target candidates in numerous cancers, including those of the lung and colorectum (Cataisson et al., 2016; Gou et al., 2016; Misale et al., 2014; Pietrantonio et al., 2016; Shida et al., 2004; Takahashi et al., 2016). In metastatic colorectal cancer, amplification and overexpression of MET is a key contributing factor to resistance to anti-EGFR therapies (Misale et al., 2014; Takahashi et al., 2016). Furthermore, dual inhibition of MET and EGFR by "biseptic" antibodies, or combined inhibitors, demonstrated effective inhibition of tumor growth in vitro and in vivo (Castoldi et al., 2013; Lee et al., 2016a; Xu et al., 2011), in accord with our findings in GC cluster B. Recently, another dual inhibition of MET and EGFR in GC cells has emerged, based on inhibition of sphingosine 1-phosphate (S1P), a G protein-coupled receptor ligand (Shida et al., 2004).

Clusters C and D (Fig. 3), from the GSE36968 transcriptome dataset, contained both upregulated upstream and downstream WNT pathway effector genes, including WNT5A, TCF7L2, and LEF1, in accord with our previous studies (Chang et al., 2016; Nam et al., 2014). In addition, it has been found that WNT5A is an emerging druggable GC signal mediator, through transcriptional regulation by $\mathrm{HNF} 4 \alpha$ (Chang et al., 2016; Nam et al., 2014), while another study (Chang et al., 2016) indicated poor prognosis of WNT5A highexpressing patients, in particular, in Lauren-classified diffusetype GC patients. That study (Chang et al., 2016) further demonstrated WNT5A-mediated signal inhibition, through $\mathrm{HNF} 4 \alpha$ antagonism by various "rationally designed" small compounds. That finding agrees with our results in this study, showing clusters $C$ and D to associate with druggable GC signaling, as well as showing promising utility as biomarkers.

MCL analysis of PATHOME-delineated networks from GC tumor versus paired adjacent normal tissue transcriptomes (GEO: GSE27342) resulted in the identification of $35 \mathrm{NCs}$. The largest cluster (cluster F in Fig. 3) showed upregulation of multiple kinase genes (Fleuren et al., 2016), including PRKCI, INSR, RPS6KA1, CDK4, and MAPK1 (encoding the mitogen ERK2). These five gene products associate with the receptor tyrosine kinase pathways PI3KmTOR and MAPK, mediating the cell cycle and numerous core cellular processes and pathways (Fleuren et al., 2016).

Moreover, since "omics" profiling of kinases revealed context-specific functions, according to cancer tissue types (Fleuren et al., 2016), cluster F may associate with specific GC tumor-oriented kinome characteristics. Also, inhibitors of kinases found upregulated in this cluster could be repurposed, from other cancers, to GC. For example, the signal transducers, RPS6KA1 and MAPK1, found in cluster F, also associate with other cancer tissue types (Kyriakis and Avruch, 2001; Lara et al., 2011; Salhi et al., 2015; Zhao et al., 1995). Of these, ERK2 (encoded by the gene $M A P K 1$ ) was reported to increase the activity and phosphorylation of the oncoprotein RPS6KA1 (ribosomal protein S6 kinase, $90 \mathrm{kDa}$, polypeptide A1), leading to cancer cell proliferation (Kyriakis and Avruch, 2001; Zhao et al., 1995). RPS6KA1 has also been recognized as a therapeutic target in lung cancer (Lara et al., 2011) and nodular type melanoma (Salhi et al., 2015).

NCs E and G (Fig. 3), derived from the GSE27342 dataset (GC tumors vs. adjacent normal tissues), revealed involvement of the mitogenic Syk/Lyn and JAK/STAT pathways, respectively. Specifically, the spleen tyrosine kinase (SYK, 
A
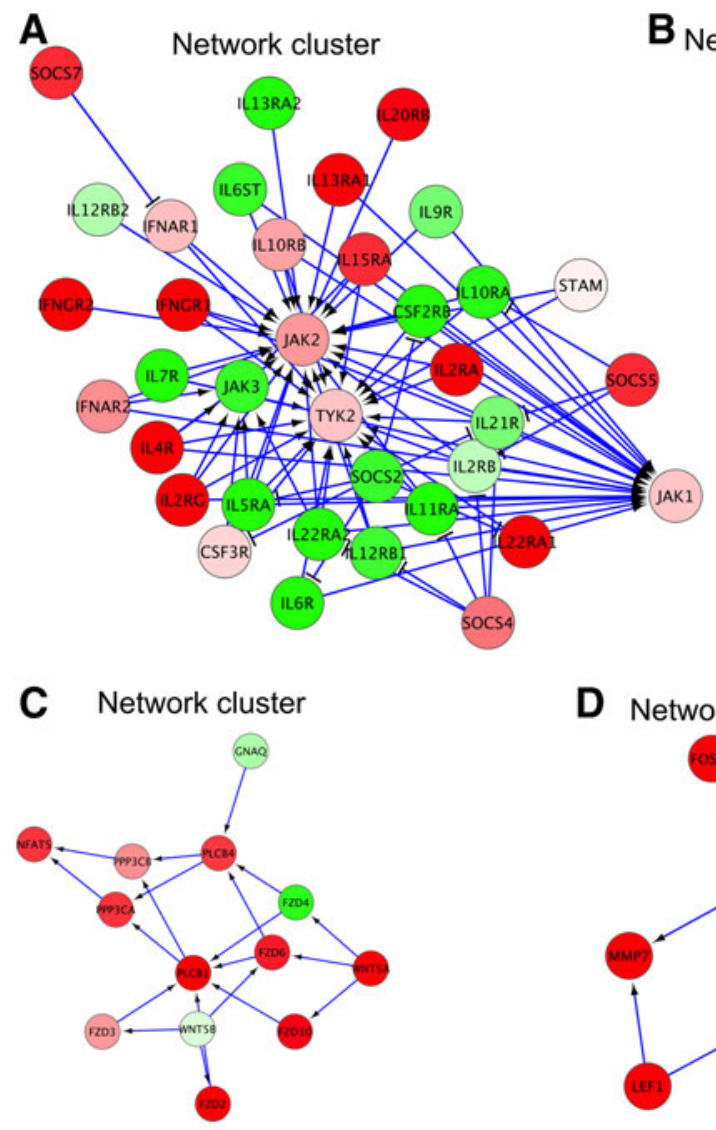

F Network cluster

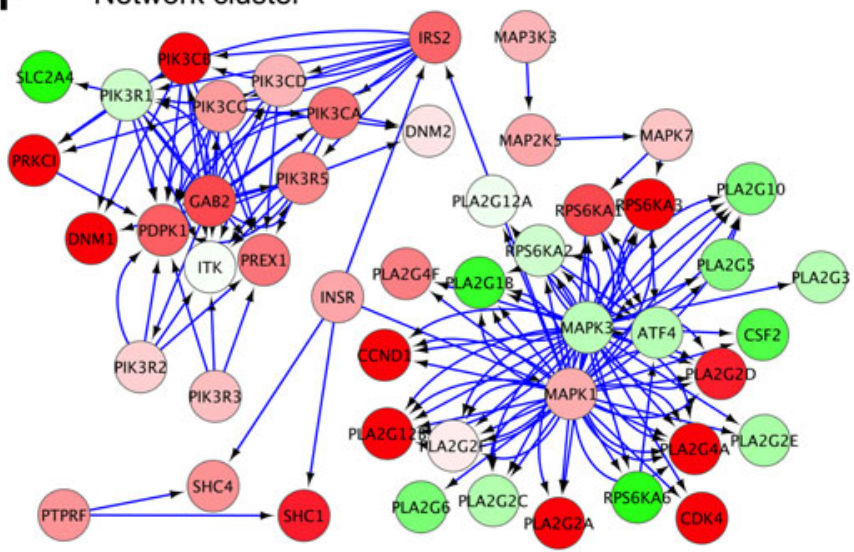

D Network cluster

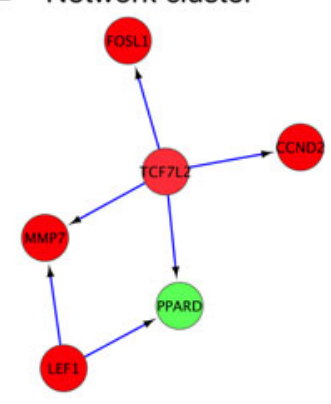

E

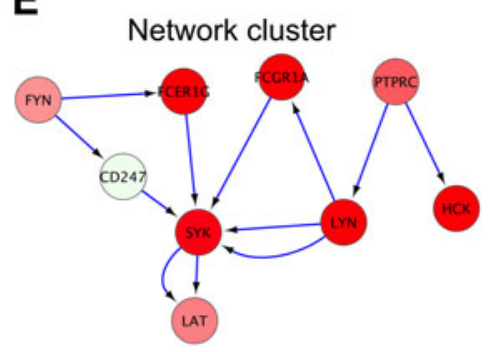

G Network cluster

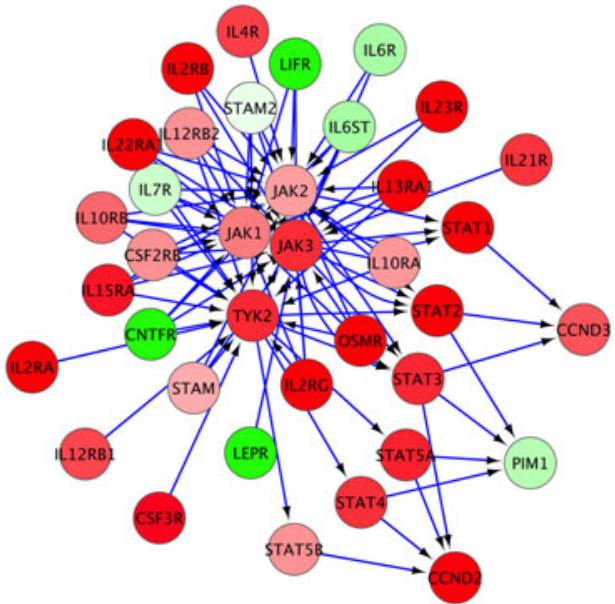

FIG. 3. Several selected NCs from the GC datasets by PATHOME network-derived network clustering method. NCs A-D were derived from GSE36968, and NCs E-G were obtained from GSE27342. The red node colors indicate upregulated genes in GC tumors (compared to normal tissue), while the green node colors represent downregulated genes.

in cluster E), expressed in majority of hematopoietic cells, has been recognized as a therapeutic target in autoimmune diseases such as rheumatoid arthritis (Geahlen, 2014).

In addition, SYK has been recently recognized as a prosurvival factor in breast cancers, as well as hematopoietic malignancies (Lee et al., 2016b), representing a strong candidate for anticancer therapy (Geahlen, 2014). In fact, fostamatinib, cerdulatinib, and entospletinib, as Syk inhibitors, are under clinical investigation as single or adjuvant agents, in certain types of leukemia (Coffey et al., 2014; Sharman et al., 2015). LAT (Linker For Activation Of T-Cells), in this same cluster, is a downstream effector of Syk signaling, and in vitro $L A T$ knockdown decreased proliferation, as well as migration, of GC cell lines (Wang et al., 2013).

Moreover, cluster $\mathrm{E}$ revealed a potentially druggable GC target, including possible "repurposing" of cerdulatinib, a dual inhibitor of Syk and JAK/STAT signaling in certain types of diffuse large B cell lymphoma cell lines (Ma et al., 2015). This suggests likely GC association with NC G, also consisting JAK/STAT signaling. Therefore, a synergistic 
effect, by downregulation of NCs E and G, should be established for developing new GC therapeutic strategies.

We also identified the consensus genes of the PATHOMENCs among the three datasets. Restricting to PATHOMENCs containing greater than or equal to five genes, we compared the genes for the PATHOME-NCs between the three datasets. As a result, we found 153 consensus genes (Supplementary Fig. S2). Out of the 153 genes (Supplementary Table S4), EGFR, JAK2, JAK3, MAPK1, TYK2, and WNT5A resided in the aforementioned NCs. Again, those genes associated with JAK/STAT as well as WNT signaling.

\section{Conclusions}

The use of PATHOME-NCs showed better relevance to cancer, in agreement with a reference set of cancer-functional contexts, compared to ARACNE-NCs. The JAK/STAT signaling, in GC PATHOME-NCs, has now been revealed in the pathogenesis of many diverse cancer types, holding promise for the application of effective targeted GC therapies or personalized medicine in this devastating type of cancer. Furthermore, rigorous analytical comparisons (Abu-Asab et al., 2008) and experimental validation are necessary to advance this line of research on cancer transcriptome data analysis.

\section{Acknowledgments}

This research was supported by the Gachon University Gil Medical Center (Grant number: 2016-06) and Basic Science Research Program, through the National Research Foundation of Korea (NRF), funded by the Ministry of Education (NRF-2016R1D1A1B03933145) to S.N. The author thanks Curt Balch for English editing.

\section{Author Disclosure Statement} exist.

The author declares that no conflicting financial interests

\section{References}

Abu-Asab MS, Chaouchi M, and Amri H. (2008). Phylogenetic modeling of heterogeneous gene-expression microarray data from cancerous specimens. OMICS 12, 183-199.

Assenov Y, Ramirez F, Schelhorn SE, Lengauer T, and Albrecht M. (2008). Computing topological parameters of biological networks. Bioinformatics 24, 282-284.

Aytes A, Mitrofanova A, Lefebvre C, et al. (2014). Crossspecies regulatory network analysis identifies a synergistic interaction between FOXM1 and CENPF that drives prostate cancer malignancy. Cancer Cell 25, 638-651.

Bang YJ, Van Cutsem E, Feyereislova A, et al. (2010). Trastuzumab in combination with chemotherapy versus chemotherapy alone for treatment of HER2-positive advanced gastric or gastro-oesophageal junction cancer (ToGA): A phase 3, open-label, randomised controlled trial. Lancet 376, 687-697.

Barabasi AL, and Oltvai ZN. (2004). Network biology: Understanding the cell's functional organization. Nat Rev Genet $5,101-113$.

Berger SI, and Iyengar R. (2009). Network analyses in systems pharmacology. Bioinformatics 25, 2466-2472.

Castoldi R, Ecker V, Wiehle L, et al. (2013). A novel bispecific EGFR/Met antibody blocks tumor-promoting phe- notypic effects induced by resistance to EGFR inhibition and has potent antitumor activity. Oncogene 32, 55935601.

Cataisson C, Michalowski AM, Shibuya K, et al. (2016). MET signaling in keratinocytes activates EGFR and initiates squamous carcinogenesis. Sci Signal 9, ra62.

Chang HR, Nam S, Kook MC, et al. (2016). HNF4alpha is a therapeutic target that links AMPK to WNT signalling in early-stage gastric cancer. Gut $65,19-32$.

Coffey G, Betz A, DeGuzman F, et al. (2014). The novel kinase inhibitor PRT062070 (Cerdulatinib) demonstrates efficacy in models of autoimmunity and B-cell cancer. J Pharmacol Exp Ther 351, 538-548.

Cui J, Chen Y, Chou WC, et al. (2011). An integrated transcriptomic and computational analysis for biomarker identification in gastric cancer. Nucleic Acids Res 39, 1197-1207.

Doncheva NT, Assenov Y, Domingues FS, and Albrecht M. (2012). Topological analysis and interactive visualization of biological networks and protein structures. Nat Protoc 7, 670-685.

Dunn GP, Koebel CM, and Schreiber RD. (2006). Interferons, immunity and cancer immunoediting. Nat Rev Immunol 6, 836-848.

Ferrara N, and Adamis AP. (2016). Ten years of anti-vascular endothelial growth factor therapy. Nat Rev Drug Discov 15, 385-403.

Fleuren ED, Zhang L, Wu J, and Daly RJ. (2016). The kinome 'at large' in cancer. Nat Rev Cancer 16, 83-98.

Futreal PA, Coin L, Marshall M, et al. (2004). A census of human cancer genes. Nat Rev Cancer 4, 177-183.

Geahlen RL. (2014). Getting Syk: Spleen tyrosine kinase as a therapeutic target. Trends Pharmacol Sci 35, 414-422.

Goel HL, and Mercurio AM. (2013). VEGF targets the tumour cell. Nat Rev Cancer 13, 871-882.

Gou LY, Li AN, Yang JJ, et al. (2016). The coexistence of MET over-expression and an EGFR T790M mutation is related to acquired resistance to EGFR tyrosine kinase inhibitors in advanced non-small cell lung cancer. Oncotarget 7, 51311-51319.

Goymer P. (2008). Network biology: Why do we need hubs? Nat Rev Genet 9, 650.

Hanahan D, and Weinberg RA. (2011). Hallmarks of cancer: The next generation. Cell 144, 646-674.

Huang DW, Sherman BT, Tan Q, et al. (2007). The DAVID Gene Functional Classification Tool: A novel biological module-centric algorithm to functionally analyze large gene lists. Genome Biol 8, R183.

Jia J, Zhu F, Ma X, Cao Z, Li Y, and Chen YZ. (2009). Mechanisms of drug combinations: Interaction and network perspectives. Nat Rev Drug Discov 8, 111-128.

Karagoz K, Sinha R, and Arga KY. (2015). Triple negative breast cancer: A multi-omics network discovery strategy for candidate targets and driving pathways. OMICS 19, 115-130.

Kim YH, Liang H, Liu X, et al. (2012). AMPKalpha modulation in cancer progression: Multilayer integrative analysis of the whole transcriptome in Asian gastric cancer. Cancer Res 72, 2512-2521.

Kyriakis JM, and Avruch J. (2001). Mammalian mitogenactivated protein kinase signal transduction pathways activated by stress and inflammation. Physiol Rev 81, 807-869.

Lara R, Mauri FA, Taylor H, et al. (2011). An siRNA screen identifies RSK1 as a key modulator of lung cancer metastasis. Oncogene 30, 3513-3521.

Lee BS, Kim HJ, Hwang JW, et al. (2016a). The dual inhibition of Met and EGFR by ME22S, a novel Met/EGFR bispecific monoclonal antibody, suppresses the proliferation and invasion of laryngeal cancer. Ann Surg Oncol 23, 2046-2053. 
Lee SJ, Choi JS, Han BG, et al. (2016b). Crystal structures of spleen tyrosine kinase in complex with novel inhibitors: Structural insights for design of anticancer drugs. FEBS J 283, 3613-3625.

Ma J, Xing W, Coffey G, et al. (2015). Cerdulatinib, a novel dual SYK/JAK kinase inhibitor, has broad anti-tumor activity in both $\mathrm{ABC}$ and GCB types of diffuse large B cell lymphoma. Oncotarget 6, 43881-43896.

Margolin AA, Nemenman I, Basso K, et al. (2006). ARACNE: An algorithm for the reconstruction of gene regulatory networks in a mammalian cellular context. BMC Bioinformatics 7 Suppl 1, S7.

Matsueda S, and Graham DY. (2014). Immunotherapy in gastric cancer. World J Gastroenterol 20, 1657-1666.

Mirsafian H, Ripen AM, Manaharan T, Mohamad SB, and Merican AF. (2016). Toward a reference gene catalog of human primary monocytes. OMICS 20, 627-634.

Misale S, Di Nicolantonio F, Sartore-Bianchi A, Siena S, and Bardelli A. (2014). Resistance to anti-EGFR therapy in colorectal cancer: From heterogeneity to convergent evolution. Cancer Discov 4, 1269-1280.

Morris JH, Apeltsin L, Newman AM, et al. (2011). clusterMaker: A multi-algorithm clustering plugin for Cytoscape. BMC Bioinformatics 12, 436.

Nam S, Chang HR, Kim KT, et al. (2014). PATHOME: An algorithm for accurately detecting differentially expressed subpathways. Oncogene 33, 4941-4951.

Nam S, Long X, Kwon C, Kim S, and Nephew KP. (2012). An integrative analysis of cellular contexts, miRNAs and mRNAs reveals network clusters associated with antiestrogen-resistant breast cancer cells. BMC Genomics 13, 732.

Nam S, and Park T. (2012). Pathway-based evaluation in early onset colorectal cancer suggests focal adhesion and immunosuppression along with epithelial-mesenchymal transition. PLoS One 7, e31685.

Ohaegbulam KC, Assal A, Lazar-Molnar E, Yao Y, and Zang X. (2015). Human cancer immunotherapy with antibodies to the PD-1 and PD-L1 pathway. Trends Mol Med 21, 24-33.

Pietrantonio F, Oddo D, Gloghini A, et al. (2016). MET-driven resistance to dual EGFR and BRAF blockade may be overcome by switching from EGFR to MET inhibition in BRAF mutated colorectal cancer. Cancer Discov 6, 963-971.

Rane SG, and Reddy EP. (2000). Janus kinases: Components of multiple signaling pathways. Oncogene 19, 5662-5679.

Ritprajak P, and Azuma M. (2015). Intrinsic and extrinsic control of expression of the immunoregulatory molecule PDL1 in epithelial cells and squamous cell carcinoma. Oral Oncol 51, 221-228.

Salhi A, Farhadian JA, Giles KM, et al. (2015). RSK1 activation promotes invasion in nodular melanoma. Am J Pathol 185, 704-716.

Sharman J, Hawkins M, Kolibaba K, et al. (2015). An openlabel phase 2 trial of entospletinib (GS-9973), a selective spleen tyrosine kinase inhibitor, in chronic lymphocytic leukemia. Blood 125, 2336-2343.

Shida D, Kitayama J, Yamaguchi H, et al. (2004). Sphingosine 1-phosphate transactivates c-Met as well as epidermal growth factor receptor (EGFR) in human gastric cancer cells. FEBS Lett 577, 333-338.
Slattery ML, Lundgreen A, and Wolff RK. (2014). VEGFA, FLT1, KDR and colorectal cancer: Assessment of disease risk, tumor molecular phenotype, and survival. Mol Carcinog 53 Suppl 1, E140-E150.

Takahashi N, Iwasa S, Taniguchi H, et al. (2016). Prognostic role of ERBB2, MET and VEGFA expression in metastatic colorectal cancer patients treated with anti-EGFR antibodies. Br J Cancer 114, 1003-1011.

Torre LA, Bray F, Siegel RL, Ferlay J, Lortet-Tieulent J, and Jemal A. (2015). Global cancer statistics, 2012. CA Cancer J Clin 65, 87-108.

Ubel C, Mousset S, Trufa D, Sirbu H, and Finotto S. (2013). Establishing the role of tyrosine kinase 2 in cancer. Oncoimmunology 2, e22840.

Waldron L, and Riester M. (2016). Meta-analysis in gene expression studies. Methods Mol Biol 1418, 161-176.

Wang J, Chen X, Su L, Li P, Liu B, and Zhu Z. (2013). LAT-1 functions as a promotor in gastric cancer associated with clinicopathologic features. Biomed Pharmacother 67, 693-699.

Wu Y, Grabsch H, Ivanova T, et al. (2013). Comprehensive genomic meta-analysis identifies intra-tumoural stroma as a predictor of survival in patients with gastric cancer. Gut 62, 1100-1111.

$\mathrm{Xu}$ H, Stabile LP, Gubish CT, Gooding WE, Grandis JR, and Siegfried JM. (2011). Dual blockade of EGFR and c-Met abrogates redundant signaling and proliferation in head and neck carcinoma cells. Clin Cancer Res 17, 4425-4438.

Zhao Y, Bjorbaek C, Weremowicz S, Morton CC, and Moller DE. (1995). RSK3 encodes a novel pp90rsk isoform with a unique $\mathrm{N}$-terminal sequence: Growth factor-stimulated kinase function and nuclear translocation. Mol Cell Biol 15, 4353-4363.

Address correspondence to: Seungyoon Nam, PhD

Department of Genome Medicine and Science College of Medicine Gachon University 774 Beongil 21 Namdongdae-ro Namdong-gu Incheon 21565

Korea

E-mail: nams@gachon.ac.kr

$\begin{aligned} & \text { Abbreviations Used } \\ \text { ARACNE }= & \text { Algorithm for the Reconstruction } \\ & \text { of Accurate Cellular Networks } \\ \mathrm{GC} & =\text { gastric cancer } \\ \mathrm{GO} & =\text { Gene Ontology } \\ \mathrm{GRNs} & =\text { gene regulatory networks } \\ \mathrm{LAT} & =\text { Linker For Activation Of T Cells } \\ \mathrm{MCL} & =\text { Markov Clustering algorithm } \\ \mathrm{NC} & =\text { network cluster } \\ \text { PATHOME } & =\text { pathway and transcriptome information } \\ \mathrm{SPNS} & =\text { signaling pathway networks } \\ \mathrm{SYK} & =\text { spleen tyrosine kinase }\end{aligned}$

